\title{
Preoperative nutritional risk index and postoperative one-year skeletal muscle loss can predict the prognosis of patients with gastric adenocarcinoma: a registry-based study
}

Kyung Won Kim ${ }^{1,2+}$, Koeun Lee ${ }^{1,3 \dagger}$, Jung-Bok Lee ${ }^{4}$, Taeyong Park ${ }^{5}$, Seungwoo Khang ${ }^{5}$, Heeryeol Jeong ${ }^{5}$, Chang-Seok Ko' , Jeong-Hwan Yook ${ }^{1}$, Byung-Sik Kim ${ }^{1}$ and In-Seob Lee ${ }^{1 *}$ (1)

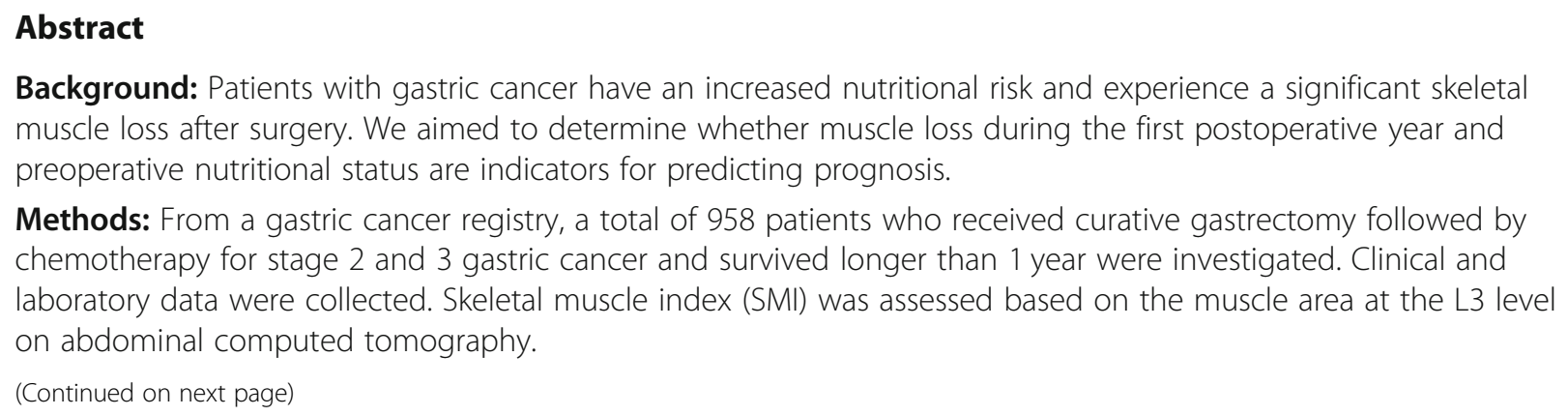
muscle loss after surgery. We aimed to determine whether muscle loss during the first postoperative year and preoperative nutritional status are indicators for predicting prognosis.

Methods: From a gastric cancer registry, a total of 958 patients who received curative gastrectomy followed by chemotherapy for stage 2 and 3 gastric cancer and survived longer than 1 year were investigated. Clinical and laboratory data were collected. Skeletal muscle index (SMI) was assessed based on the muscle area at the L3 level on abdominal computed tomography.

(Continued on next page)

\footnotetext{
* Correspondence: inseoblee77@amc.seoul.kr

${ }^{\dagger}$ Kyung Won Kim and Koeun Lee equally contributed to this work as co-first authors.

${ }^{1}$ Department of Surgery, Gastric Cancer Center, Asan Medical Center,

University of Ulsan College of Medicine, 88, Olympic-ro 43-gil, Songpa-gu, Seoul 05505, South Korea

Full list of author information is available at the end of the article
}

(c) The Author(s). 2021 Open Access This article is licensed under a Creative Commons Attribution 4.0 International License, which permits use, sharing, adaptation, distribution and reproduction in any medium or format, as long as you give appropriate credit to the original author(s) and the source, provide a link to the Creative Commons licence, and indicate if changes were made. The images or other third party material in this article are included in the article's Creative Commons licence, unless indicated otherwise in a credit line to the material. If material is not included in the article's Creative Commons licence and your intended use is not permitted by statutory regulation or exceeds the permitted use, you will need to obtain permission directly from the copyright holder. To view a copy of this licence, visit http://creativecommons.org/licenses/by/4.0/ The Creative Commons Public Domain Dedication waiver (http://creativecommons.org/publicdomain/zero/1.0/) applies to the data made available in this article, unless otherwise stated in a credit line to the data. 


\begin{abstract}
(Continued from previous page)
Results: Preoperative nutritional risk index (NRI) and postoperative decrement of SMI (dSMI) were significantly associated with overall survival (hazards ratio: 0.976 [95\% Cl: 0.962-0.991] and 1.060 [95\% Cl: 1.035-1.085], respectively) in a multivariate Cox regression analysis. Recurrence, tumor stage, comorbidity index were also significant prognostic indicators. Kaplan-Meier analyses exhibited that patients with higher NRI had a significantly longer survival than those with lower NRI (5-year overall survival: $75.8 \%$ vs. $63.0 \%, P<0.001$ ). In addition, a significantly better prognosis was observed in a patient group with less decrease of SMI (5-year overall survival: $75.7 \%$ vs. $66.2 \%, P=0.009$ ). A logistic regression analysis demonstrated that the performance of preoperative NRI and $\mathrm{dSMI}$ in mortality prediction was quite significant (AUC: $0.63, P<0.001$ ) and the combination of clinical factors enhanced the predictive accuracy to the AUC of $0.90(P<0.001)$. This prognostic relevance of NRI and dSMI was maintained in patients experiencing tumor recurrence and highlighted in those with stage 3 gastric adenocarcinoma.

Conclusions: Preoperative NRI is a predictor of overall survival in stage 2 or 3 gastric cancer patients and skeletal muscle loss during the first postoperative year was significantly associated with the prognosis regardless of relapse in stage 3 tumors. These factors could be valuable adjuncts for accurate prediction of prognosis in gastric cancer patients.
\end{abstract}

Keywords: Gastric cancer, Surgery, Muscle loss, Nutrition, Prognosis, Survival, Sarcopenia

\section{Background}

Surgery is the mainstay of curative treatment for gastric cancer. Gastrectomy induces physiologic derangements including worsened nutritional status, significant weight loss, and decreased muscle and fat volume. The loss of stomach reservoir function, rapid intestinal transit time, and foods bypassing the proximal small intestine are responsible for these postoperative changes [1-3].

In the first year after gastrectomy, most patients experience dramatic physiologic changes, like cascade of sarcopenia and malnutrition. Indeed, our prior study demonstrated that skeletal muscle mass and nutritional parameters decrease sharply for the first 3 months and slowly decrease for the remaining 9 months, eventually leading to a loss of $8 \sim 15 \%$ of the initial body weight and $3 \sim 5 \%$ of the muscle area [3]. In addition, adjuvant chemotherapy, which is the standard treatment in stage 2 and 3 gastric cancer, exacerbates skeletal muscle loss and nutritional status of patients [4].

Several tumor factors including the TNM staging system are powerful predictors of survival in gastric cancer patients treated with surgery; however, it is insufficient in reflecting the heterogeneity of the clinical course. A number of patient variables such as age, performance status, and neutrophil/lymphocyte ratio have been identified as prognostic factors for overall survival in the last decade [5-7]. Recently, the prognostic value of preoperative sarcopenia and nutritional status have been gaining emphasis $[8,9]$. However, we have been impressed that progressive skeletal muscle loss after gastrectomy might also be a powerful prognostic indicator based on thousands of cases of practice in our dedicated gastric cancer center. Especially, it seems that the muscle loss at 1 year after surgery, when adjuvant chemotherapy is completed, might be particularly important to indicate overall health state and prognosis of patients.

Thus, we hypothesized that the progressive muscle loss during the first postoperative year is a significant predictor of overall survival along with preoperative nutritional status in patients with stage 2 or 3 gastric cancer who survived longer than 1 year. To evaluate our hypothesis, we performed this large-scale study with the aid of artificial intelligence techniques to measure body composition.

\section{Methods}

The protocol for this retrospective and registry-based cohort study was approved by the institutional review board of Asan Medical Center, Seoul, Korea (IRB No. 2017-0216). This study was reported according to the Transparent Reporting of a multivariable prediction model for Individual Prognosis or Diagnosis (TRIPOD) guidelines [10].

\section{Patient selection}

The current study was conducted on the basis of a comprehensive and prospectively built gastric cancer surgery registry containing the demographic characteristics of patients, preoperative evaluation results, surgery-related and postoperative outcomes, pathologic information, and follow-up data. From the registry, the data of 9940 patients who received surgery for biopsy-proven primary gastric adenocarcinoma from 2007 to 2012 at Asan Medical Center, Seoul, Korea were initially extracted. Subsequently, we included patients based on specific inclusion and exclusion criteria.

The inclusion criteria were as follows: (a) patients who were treated with curative gastrectomy followed by 
adjuvant chemotherapy using S-1 or capecitabine plus oxaliplatin (XELOX) for pathologic stage 2 and 3 gastric cancer based on the American Joint Committee on Cancer 7th edition [11], (b) patients aged between 18 and 85 years, (c) patients who abide by a regular follow-up protocol with available data on demographic measures, laboratory findings, and abdominopelvic computed tomography (CT) images. Patients (a) who died within 1 year after surgery, (b) who received neoadjuvant treatment, (c) with a history of previous partial gastrectomy, (d) with any synchronous malignancy in another organ, and (e) with inappropriate clinical or radiologic data were excluded. The patient selection process is illustrated in Fig. 1.

\section{Medical data collection and follow-up}

The preoperative clinicopathologic characteristics of the patients, including age, sex, body weight, height, body mass index (BMI), and history of any synchronous malignancy or comorbidity, were evaluated. Comorbidity was assessed by Charlson Comorbidity Index [12]. Information about the type of operation (open vs. laparoscopic approach), type of gastrectomy (distal vs. total gastrectomy), extent of lymph node dissection (D1 vs. D1+ vs. D2), and pathologic data including Lauren's classification [13] and pathologic tumor stage were collected from the registry.

Laboratory parameters such as serum protein and albumin at preoperative and postoperative periods were extracted from the registry. The nutritional risk index (NRI) was calculated based on the formula $(1.519 \times$ serum albumin $\mathrm{g} / \mathrm{L})+0.417 \times$ (present weight / usual weight $\times 100$ [14]. The difference in NRI (dNRI) between before and 1 year after surgery was assessed. Body weight and BMI were measured at all visits. Recurrence was evaluated by serum tumor marker measurement, endoscopy, and abdominopelvic CT following the guidelines for gastric cancer treatment [15].

\section{Assessment of body composition}

CT scans obtained before and 1 year after surgery were selected for body morphometric analysis. Preoperative CT was checked within 1 month before surgery on average. Body composition was assessed with abdominopelvic CT using an automated artificial intelligence software (AID-U ${ }^{\mathrm{rm}}$, iAID Inc., Seoul, Korea), which was developed using a fully convolutional network (FCN) segmentation technique [16]. A specialized abdominal radiologist (K.W.K), who was blinded to the clinical information, selected the axial CT slice at the L3 vertebral inferior endplate level in a semi-automatic manner with the aid of coronal reconstructed images. Then, the chosen images were automatically segmented to generate the boundary of total abdominal muscles. The skeletal muscle area (SMA) including all muscles on the selected axial images, i.e., psoas, paraspinal, transversus abdominis, rectus abdominis, quadratus lumborum, and internal and external obliques, were demarcated using predetermined thresholds $(-29$ to +150 Hounsfield units). The visceral fat area (VFA) and subcutaneous fat area (SFA) were also demarcated using fat tissue thresholds ( -190 to -30 Hounsfield units) (Supplementary Figure S1). The SMA was adjusted for the square of the height (SMA/height ${ }^{2}$ ), which is referred to as the skeletal

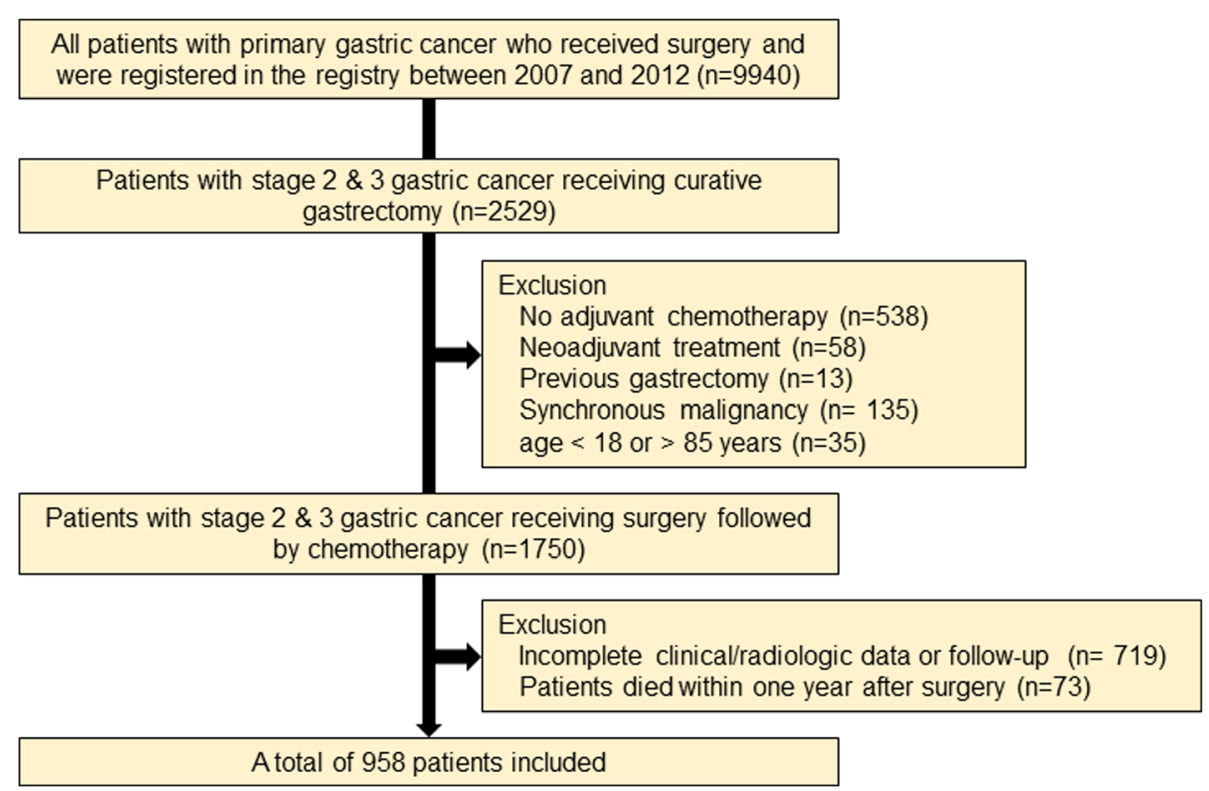

Fig. 1 Flow chart of the patient selection 
muscle index (SMI) [17]. The differences in SMA and SMI between before and 1 year after surgery were calculated to yield dSMA and dSMI, respectively.

\section{Statistical analysis}

Continuous data were reported as means with standard deviations, and categorical data were presented as proportions. Normality was assessed using frequency histograms and the Kolmogorov-Smirnov test. A paired t-test was used to compare preoperative with postoperative body composition and nutritional parameters. Overall survival was defined as the time interval between the date of surgery and the date of patient's death from any cause. Patients were censored at 5 years for overall survival if they were alive at 5 years after surgery. Follow-up loss and recurrence were also regarded as censoring.

The primary endpoint of this study was to determine prognostic factors to predict overall survival in patients who lived longer than 1 year after gastrectomy. A Cox proportional hazards model was used for univariate and multivariate analyses, and outcomes were reported as hazards ratios and $95 \%$ confidence intervals. The covariates that were significant in univariate Cox analysis were included in multivariate one. In multivariate analysis, the backward elimination method was used to fit the model and to determine the final significant predictors after confirming that there was no significant multicollinearity between variables by examining the correlation matrices.

The secondary endpoint was to evaluate predictive performance of the progressive muscle loss (dSMI) and preoperative nutritional status (NRI) to predicting mortality. Patients were dichotomized (high-risk vs. low-risk group) according to the median value of dSMI and preoperative NRI. Survival curves were estimated for each group using the Kaplan-Meier method and compared statistically using the log rank test. Logistic regression analysis with Enter model was carried out to assess the performance dSMI and NRI for prediction of patient mortality during the follow-up period, and the performance was evaluated with receiver operating characteristic (ROC) curves and area under the curves (AUCs).

As a subgroup analysis, all these statistical analyses were performed in a recurrence group and additional analyses were also undertaken according to tumor stage. $P$-value of $<0.05$ was used as the level of significance for the study. Statistical analyses were performed using SAS software version 9.4 (SAS Institute Inc., Cary, NC, USA) and IBM SPSS ${ }^{\bullet}$ version 26 (IBM, Armonk, NY, USA).

\section{Results}

Baseline characteristics

Figure 1 illustrates the patient selection process. Among the initial 9940 patients who were registered in the gastric cancer surgery registry, there were 1750 patients with stage 2 or 3 gastric adenocarcinoma who were treated with gastrectomy followed by adjuvant chemotherapy. During the process, we excluded 538 patients not receiving adjuvant treatment including cases with tumor stage of pT1N+ or pT3N0 $(n=455)$ which are not indicated to adjuvant treatment according to Korean and Japanese gastric cancer treatment guidelines, and those with poor general condition, severe comorbidity, and postoperative complication $(n=83)$.

After excluding those who had insufficient image quality for body morphometry analysis or incomplete followup data $(n=719)$ and who died within 1 year after surgery $(n=73)$, a total of 958 patients were included in the study. As seen in Table 1, we divided patients into a recurrence group $(n=293)$ and a non-recurrence group $(n=665)$, because the treatment and prognosis of two population differ significantly. In a recurrence group, 26 patients experienced tumor relapse within 6 months after surgery. Type of gastrectomy and distribution of tumor stage were different between two groups; total gastrectomy and tumors with an advanced stage were more frequently observed in patients experiencing relapse. The other variables did not differ significantly between the two groups.

Among patients, $42.4 \%$ had at least one comorbidity and $89.4 \%$ received open surgery. Distal gastrectomy was more frequently performed than total gastrectomy (57.2\% versus $42.8 \%)$ (Table 1$)$.

Changes in body composition and nutritional parameters Body weight diminished during the first postoperative year (mean loss of $5.9 \mathrm{~kg}$ ). All parameters related to body composition (SMA, SMI, SFA, VFA) and NRI measured at 1 year after surgery showed a significant decrement compared with preoperative values as well (Supplementary Table S1). These changes were consistently observed in both the recurrence group and the nonrecurrence group. The representative cases were presented in Supplementary Figure S2.

\section{Prognostic relevance of skeletal muscle loss and NRI}

Univariate and multivariate cox-hazard regression analysis results were presented in Table 2 . In univariate analysis, the recurrence was the strongest prognostic indicator (HR 13.992). Among the clinicopathologic variables, old age, male sex, higher comorbidity index, advanced tumor stage, open surgery, total gastrectomy, and larger tumor size were associated with a shorter survival period in patients with stage 2 and 3 gastric cancer. Among preoperative body composition and nutrition related parameters, SFA and NRI affected prognosis. Among the parameters for body composition and nutrition change between before and 1 year after surgery, dSMI was predictive of overall survival. 
Table 1 Clinicopathologic characteristics of patients with stage 2 and 3 gastric cancer

\begin{tabular}{|c|c|c|c|c|}
\hline Variables & Whole patients $(N=958)$ & Recurrence group $(N=293)$ & Non-recurrence group $(N=665$ ) & $P$-value \\
\hline Mean age at operation, years $( \pm S D)$ & $57.0( \pm 12.3)$ & $57.7( \pm 12.6)$ & $56.7( \pm 12.2)$ & 0.240 \\
\hline Sex & & & & 0.449 \\
\hline Male & $624(65.1 \%)$ & $196(66.9 \%)$ & $428(64.4 \%)$ & \\
\hline Female & $334(34.9 \%)$ & $97(33.1 \%)$ & $237(35.6 \%)$ & \\
\hline Comorbidity & & & & 0.763 \\
\hline Diabetes & $138(14.4 \%)$ & $44(15.0 \%)$ & $94(14.1 \%)$ & \\
\hline Hypertension & $271(28.3 \%)$ & $86(29.4 \%)$ & $185(27.8 \%)$ & \\
\hline Lung disease & $38(4.0 \%)$ & $14(4.8 \%)$ & $24(3.6 \%)$ & \\
\hline Cerebrovascular accident & $64(6.7 \%)$ & $20(6.8 \%)$ & $44(6.6 \%)$ & \\
\hline Heart disease & $25(2.6 \%)$ & $8(2.7 \%)$ & $17(2.6 \%)$ & \\
\hline Liver cirrhosis & $9(0.9 \%)$ & $4(1.4 \%)$ & $5(0.7 \%)$ & \\
\hline Renal disease & $15(1.6 \%)$ & $4(1.4 \%)$ & $11(1.7 \%)$ & \\
\hline Charlson Comorbidity Index & & & & 0.065 \\
\hline 0 & $243(24.4 \%)$ & $62(21.1 \%)$ & $181(27.2 \%)$ & \\
\hline $1-2$ & $465(48.5 \%)$ & $147(50.2 \%)$ & $318(47.8 \%)$ & \\
\hline $3-6$ & $250(26.1 \%)$ & $84(28.7 \%)$ & $166(25.0 \%)$ & \\
\hline Type of operation & & & & 0.063 \\
\hline Laparoscopy & $102(10.6 \%)$ & $23(7.8 \%)$ & $79(11.9 \%)$ & \\
\hline Open & $856(89.4 \%)$ & $270(92.2 \%)$ & $586(88.1 \%)$ & \\
\hline Type of gastrectomy & & & & 0.007 \\
\hline Distal gastrectomy & $549(57.2 \%)$ & $149(50.9 \%)$ & $400(60.2 \%)$ & \\
\hline Total gastrectomy & $409(42.8 \%)$ & $144(49.1 \%)$ & $265(39.8 \%)$ & \\
\hline Extent of lymphadenectomy & & & & 0.934 \\
\hline D1 & $20(2.0 \%)$ & $6(2.0 \%)$ & $14(2.1 \%)$ & \\
\hline D1 plus & $241(25.2 \%)$ & 77 (26.3\%) & $164(24.7 \%)$ & \\
\hline D2 & $697(72.8 \%)$ & $210(71.7 \%)$ & $487(73.2 \%)$ & \\
\hline TNM stage $^{a}$ & & & & $<0.001$ \\
\hline $2 \mathrm{~A}$ & 199 (20.8\%) & $26(8.9 \%)$ & $173(26.0 \%)$ & \\
\hline $2 \mathrm{~B}$ & $237(24.7 \%)$ & $61(20.8 \%)$ & $176(26.5 \%)$ & \\
\hline $3 \mathrm{~A}$ & $194(20.3 \%)$ & $47(16.0 \%)$ & $147(22.1 \%)$ & \\
\hline $3 B$ & $194(20.3 \%)$ & $80(27.3 \%)$ & $114(17.1 \%)$ & \\
\hline $3 C$ & $134(14.0 \%)$ & 79 (27.0\%) & $55(8.3 \%)$ & \\
\hline
\end{tabular}

${ }^{\mathrm{a}}$ TNM stage was based on the American Joint Committee on Cancer 7th edition

When these significant variables were included in the multivariate analysis as covariates, the recurrence was also the strongest predictor of overall survival (HR 16.838), and comorbidity index, TNM stage, preoperative NRI, and dSMI remained as significant prognostic factors. These results support our hypothesis that progressive muscle loss during the first year after gastrectomy is an independent predictor of worse prognosis (dSMI; HR 1.060) along with protective effect of preoperative nutritional status (NRI; HR 0.976).

As tumor recurrence was a dominant factor, we separately investigated the prognostic relevance of muscle loss in the recurrence group. In univariate analysis, in addition to tumor stage, Lauren's classification, and preoperative NRI, dSMI, and dSFA were related with survival. Notably, in multivariate analysis, the dSMI (HR 1.045) and NRI (HR 0.966) maintained their significances as independent prognostic indicators of overall survival along with pathologic tumor stage and dSFA. These analyses enabled us to ascertain that dSMI and NRI were consistently associated with the prognosis irrespective of tumor recurrence. Clinicopathologic characteristics based on these two indicators were compared in Supplementary Table S2.

\section{Prognostic impact of NRI and skeletal muscle loss on stage 3 gastric adenocarcinoma}

Next, as the tumor stage was an independent prognostic factor, we further undertook a subgroup analysis by tumor stage (Table 3). A multivariate analysis exhibited that NRI was a consistent prognostic indicator for 
Table 2 Cox proportional hazards regression analyses of overall survival according to tumor recurrence

\begin{tabular}{|c|c|c|c|c|c|c|c|c|c|c|c|c|}
\hline \multirow[t]{3}{*}{ Variables } & \multicolumn{6}{|c|}{ Whole group $(N=958)$} & \multicolumn{6}{|c|}{ Recurrence group $(N=293)$} \\
\hline & \multicolumn{3}{|c|}{ Univariate analysis } & \multicolumn{3}{|c|}{ Multivariate analysis } & \multicolumn{3}{|c|}{ Univariate analysis } & \multicolumn{3}{|c|}{ Multivariate analysis } \\
\hline & $\mathrm{HR}$ & $95 \% \mathrm{Cl}$ & $P$-value & HR & $95 \% \mathrm{Cl}$ & $P$-value & HR & $95 \% \mathrm{Cl}$ & $P$-value & HR & $95 \% \mathrm{Cl}$ & $P$-value \\
\hline \multicolumn{13}{|l|}{ Clinicopathologic } \\
\hline Age (continuous) & 1.019 & $\begin{array}{l}1.011- \\
1.028\end{array}$ & $<0.001$ & & & & 1.002 & $\begin{array}{l}0.992- \\
1.012\end{array}$ & 0.701 & & & \\
\hline Sex (male) & 1.242 & $\begin{array}{l}1.007- \\
1.531\end{array}$ & 0.043 & & & & 1.009 & $\begin{array}{l}0.785- \\
1.297\end{array}$ & 0.943 & & & \\
\hline BMI (continuous) & 0.968 & $\begin{array}{l}0.935- \\
1.001\end{array}$ & 0.061 & & & & 0.999 & $\begin{array}{l}0.960- \\
1.039\end{array}$ & 0.955 & & & \\
\hline CCl (continuous) & 1.223 & $\begin{array}{l}1.138- \\
1.314\end{array}$ & $<0.001$ & 1.173 & $1.082-1.272$ & $<0.001$ & 1.068 & $\begin{array}{l}0.977- \\
1.169\end{array}$ & 0.150 & & & \\
\hline TNM stage (stage 3) & 1.927 & $\begin{array}{l}1.573- \\
2.362\end{array}$ & $<0.001$ & 1.324 & $1.054-1.663$ & 0.016 & 1.528 & $\begin{array}{l}1.175- \\
1.987\end{array}$ & 0.002 & 1.312 & $\begin{array}{l}0.998- \\
1.724\end{array}$ & 0.051 \\
\hline Operation type (open) & 1.458 & $\begin{array}{l}1.027- \\
2.072\end{array}$ & 0.035 & & & & 1.308 & $\begin{array}{l}0.845- \\
2.024\end{array}$ & 0.229 & & & \\
\hline Gastrectomy type (total) & 1.240 & $\begin{array}{l}1.020- \\
1.506\end{array}$ & 0.030 & & & & 1.109 & $\begin{array}{l}0.875- \\
1.405\end{array}$ & 0.393 & & & \\
\hline Tumor size (continuous) & 1.079 & $\begin{array}{l}1.052- \\
1.108\end{array}$ & $<0.001$ & & & & 1.022 & $\begin{array}{l}0.990- \\
1.055\end{array}$ & 0.173 & & & \\
\hline $\begin{array}{l}\text { Tumor location (upper vs. } \\
\text { others) }\end{array}$ & 1.058 & $\begin{array}{l}0.949- \\
1.181\end{array}$ & 0.310 & & & & 1.033 & $\begin{array}{l}0.921- \\
1.159\end{array}$ & 0.575 & & & \\
\hline $\begin{array}{l}\text { Lauren's classification (other } \\
\text { types vs. intestinal) }\end{array}$ & 0.929 & $\begin{array}{l}0.814- \\
1.060\end{array}$ & 0.272 & & & & 1.245 & $\begin{array}{l}1.073- \\
1.445\end{array}$ & 0.004 & 1.157 & $\begin{array}{l}0.989- \\
1.354\end{array}$ & 0.069 \\
\hline Recurrence & 13.992 & $\begin{array}{l}11.197- \\
17.486\end{array}$ & $<0.001$ & 16.838 & $13.089-21.662$ & $<0.001$ & NA & NA & NA & NA & NA & NA \\
\hline \multicolumn{13}{|c|}{ Preop. Body/Nutrition (as a continuous variable) } \\
\hline SMI & 1.000 & $\begin{array}{l}0.994- \\
1.006\end{array}$ & 0.965 & & & & 1.001 & $\begin{array}{l}0.995- \\
1.007\end{array}$ & 1.001 & & & \\
\hline SFA & 0.997 & $\begin{array}{l}0.995- \\
0.999\end{array}$ & $<0.001$ & 0.998 & $0.996-1.000$ & 0.075 & 0.999 & $\begin{array}{l}0.997- \\
1.001\end{array}$ & 0.497 & & & \\
\hline VFA & 1.000 & $\begin{array}{l}0.998- \\
1.002\end{array}$ & 0.864 & & & & 0.999 & $\begin{array}{l}0.997- \\
1.001\end{array}$ & 0.447 & & & \\
\hline NRI & 0.960 & $\begin{array}{l}0.948- \\
0.973\end{array}$ & $<0.001$ & 0.976 & $0.962-0.991$ & 0.002 & 0.981 & $\begin{array}{l}0.966- \\
0.996\end{array}$ & 0.014 & 0.966 & $\begin{array}{l}0.950- \\
0.983\end{array}$ & $<0.001$ \\
\hline \multicolumn{13}{|c|}{ Body/Nutrition change (as a continuous variable) } \\
\hline dSMl & 1.043 & $\begin{array}{l}1.019- \\
1.066\end{array}$ & $<0.001$ & 1.060 & $1.035-1.085$ & $<0.001$ & 1.045 & $\begin{array}{l}1.019- \\
1.071\end{array}$ & $<0.001$ & 1.045 & $\begin{array}{l}1.019- \\
1.073\end{array}$ & $<0.001$ \\
\hline dSFA & 1.000 & $\begin{array}{l}0.998- \\
1.003\end{array}$ & 0.816 & & & & 1.004 & $\begin{array}{l}1.002- \\
1.007\end{array}$ & 0.002 & 1.005 & $\begin{array}{l}1.002- \\
1.008\end{array}$ & 0.002 \\
\hline dVFA & 1.000 & $\begin{array}{l}0.998- \\
1.002\end{array}$ & 0.881 & & & & 1.001 & $\begin{array}{l}0.998- \\
1.003\end{array}$ & 0.660 & & & \\
\hline$d N R I$ & 1.000 & $\begin{array}{l}0.993- \\
1.006\end{array}$ & 0.917 & & & & 1.004 & $\begin{array}{l}0.995- \\
1.002\end{array}$ & 0.406 & & & \\
\hline
\end{tabular}

Abbreviations: HR Hazards ratio, CI Confidence interval, CCI Charlson comorbidity index, Preop Preoperative, SMA Skeletal muscle area, SMI Skeletal muscle index, SFA subcutaneous fat area, VFA Visceral fat area, NRI Nutritional risk index, dSMA Difference in SMA between before and one year after surgery, $d S M I$ Difference in $S M I$ between before and one year after surgery, $d N R I$ Difference in NRI between before and one year after surgery

overall survival regardless of tumor stage. Also, in patients with stage 3 gastric cancer, dSMI was significantly associated with the prognosis along with tumor recurrence, comorbidity index, and NRI in multivariate analysis. However, the statistical significance of muscle loss was not observed in stage 2 tumors.

\section{Performance of skeletal muscle loss and NRI as prognostic stratifiers}

The 5-year overall survival rate of patients included in this study were $69.4 \%$. To evaluate the role as a prognostic stratifier, patients were dichotomized (high-risk vs. low-risk group) according to the median value of 
Table 3 Cox proportional hazards regression analyses of overall survival according to tumor stage

\begin{tabular}{|c|c|c|c|c|c|c|c|c|c|c|c|c|}
\hline \multirow[t]{3}{*}{ Variables } & \multicolumn{6}{|c|}{ Stage $2(N=436)$} & \multicolumn{6}{|c|}{ Stage $3(N=522)$} \\
\hline & \multicolumn{3}{|c|}{ Univariate analysis } & \multicolumn{3}{|c|}{ Multivariate analysis } & \multicolumn{3}{|c|}{ Univariate analysis } & \multicolumn{3}{|c|}{ Multivariate analysis } \\
\hline & HR & $95 \% \mathrm{Cl}$ & $P$-value & $\mathrm{HR}$ & $95 \% \mathrm{Cl}$ & $P$-value & $\mathrm{HR}$ & $95 \% \mathrm{Cl}$ & $P$-value & HR & $95 \% \mathrm{Cl}$ & $P$-value \\
\hline \multicolumn{13}{|l|}{ Clinicopathologic } \\
\hline Age (continuous) & 1.028 & $\begin{array}{l}1.013- \\
1.044\end{array}$ & $<0.001$ & & & & 1.016 & $\begin{array}{l}1.006- \\
1.027\end{array}$ & 0.002 & & & \\
\hline Sex (male) & 1.232 & $\begin{array}{l}0.868- \\
1.749\end{array}$ & 0.243 & & & & 1.224 & $\begin{array}{l}0.943- \\
1.590\end{array}$ & 0.130 & & & \\
\hline BMI (continuous) & 0.981 & $\begin{array}{l}0.924- \\
1.041\end{array}$ & 0.516 & & & & 0.975 & $\begin{array}{l}0.935- \\
1.016\end{array}$ & 0.220 & & & \\
\hline CCl (continuous) & 1.386 & $\begin{array}{l}1.227- \\
1.566\end{array}$ & $<0.001$ & 1.154 & $1.016-1.310$ & 0.028 & 1.161 & $\begin{array}{l}1.063- \\
1.268\end{array}$ & $<0.001$ & 1.335 & $1.132-1.573$ & $<0.001$ \\
\hline Operation type (open) & 1.022 & $\begin{array}{l}0.659- \\
1.584\end{array}$ & 0.924 & & & & 1.644 & $\begin{array}{l}0.874- \\
3.093\end{array}$ & 0.123 & & & \\
\hline $\begin{array}{l}\text { Gastrectomy type } \\
\text { (total) }\end{array}$ & 1.190 & $\begin{array}{l}0.851- \\
1.664\end{array}$ & 0.309 & & & & 1.139 & $\begin{array}{l}0.895- \\
1.449\end{array}$ & 0.290 & & & \\
\hline $\begin{array}{l}\text { Tumor size } \\
\text { (continuous) }\end{array}$ & 1.075 & $\begin{array}{l}1.023- \\
1.130\end{array}$ & 0.005 & & & & 1.061 & $\begin{array}{l}1.028- \\
1.096\end{array}$ & $<0.001$ & & & \\
\hline $\begin{array}{l}\text { Tumor location (upper } \\
\text { vs. others) }\end{array}$ & 1.024 & $\begin{array}{l}0.842- \\
1.246\end{array}$ & 0.814 & & & & 1.072 & $\begin{array}{l}0.942- \\
1.220\end{array}$ & 0.293 & & & \\
\hline $\begin{array}{l}\text { Lauren's classification } \\
\text { (other types vs. } \\
\text { intestinal) }\end{array}$ & 0.865 & $\begin{array}{l}0.685- \\
1.092\end{array}$ & 0.224 & & & & 0.915 & $\begin{array}{l}0.780- \\
1.073\end{array}$ & 0.275 & & & \\
\hline Recurrence & 14.203 & $\begin{array}{l}10.027- \\
20.118\end{array}$ & $<0.001$ & 12.748 & $8.946-18.167$ & $<0.001$ & 12.486 & $\begin{array}{l}9.275- \\
16.808\end{array}$ & $<0.001$ & 17.841 & $12.542-25.378$ & $<0.001$ \\
\hline \multicolumn{13}{|c|}{ Preop. Body/Nutrition (as a continuous variable) } \\
\hline SMl & 0.998 & $\begin{array}{l}0.987- \\
1.010\end{array}$ & 0.801 & & & & 1.000 & $\begin{array}{l}0.995- \\
1.007\end{array}$ & 0.746 & & & \\
\hline SFA & 0.997 & $\begin{array}{l}0.993- \\
0.999\end{array}$ & 0.036 & 0.997 & $0.994-0.999$ & 0.044 & 0.997 & $\begin{array}{l}0.995- \\
0.999\end{array}$ & 0.008 & & & \\
\hline VFA & 1.000 & $\begin{array}{l}0.997- \\
1.003\end{array}$ & 0.961 & & & & 1.000 & $\begin{array}{l}0.998- \\
1.002\end{array}$ & 0.866 & & & \\
\hline NRI & 0.957 & $\begin{array}{l}0.936- \\
0.978\end{array}$ & $<0.001$ & 0.971 & $0.948-0.995$ & 0.019 & 0.968 & $\begin{array}{l}0.953- \\
0.983\end{array}$ & $<0.001$ & 0.973 & $0.956-0.991$ & 0.004 \\
\hline \multicolumn{13}{|c|}{ Body/Nutrition change (as a continuous variable) } \\
\hline dSMl & 1.011 & $\begin{array}{l}0.967- \\
1.057\end{array}$ & 0.619 & & & & 1.038 & $\begin{array}{l}1.013- \\
1.065\end{array}$ & 0.003 & 1.042 & $1.015-1.069$ & 0.002 \\
\hline dSFA & 1.001 & $\begin{array}{l}0.997- \\
1.005\end{array}$ & 0.536 & & & & 0.999 & $\begin{array}{l}0.996- \\
1.002\end{array}$ & 0.614 & & & \\
\hline dVFA & 1.001 & $\begin{array}{l}0.997- \\
1.005\end{array}$ & 0.518 & & & & 0.999 & $\begin{array}{l}0.996- \\
1.002\end{array}$ & 0.367 & & & \\
\hline dNRI & 1.001 & $\begin{array}{l}0.992- \\
1.011\end{array}$ & 0.784 & & & & 1.008 & $\begin{array}{l}0.998- \\
1.019\end{array}$ & 0.103 & & & \\
\hline
\end{tabular}

Abbreviations: HR Hazards ratio, $\mathrm{Cl}$ Confidence interval, $\mathrm{CCl}$ Charlson comorbidity index; Preop., preoperative, SMA Skeletal muscle area, SMI Skeletal muscle index, SFA subcutaneous fat area, VFA Visceral fat area, NRI Nutritional risk index, dSMA Difference in SMA between before and one year after surgery, dSMI Difference in SMI between before and one year after surgery, $d N R I$ Difference in NRI between before and one year after surgery

preoperative NRI (100.941) and dSMI (-2.059). KaplanMeier curves showed that patients with a better nutritional status (higher NRI) had a significantly better prognosis (5-year overall survival rate: $75.8 \%$ vs. $63.0 \%$, $P<0.001$ ) (Fig. 2a). In addition, patients with less decrease of skeletal muscle (smaller dSMI) also demonstrated longer survival period (5-year overall survival rate: $75.7 \%$ vs. $66.2 \%, P=0.009$ ) (Fig. $2 b$ ). In a subgroup analysis with the relapse group, a significant survival difference was observed between high-risk group and lowrisk group for both NRI (5-year overall survival rate: $27.9 \%$ vs. $13.7 \%, P=0.003)$ and $\mathrm{dSMI}(26.2 \%$ vs. $16.4 \%$, $P=0.006$ ) (Fig. 2c and d). Then, we conducted survival analyses based on tumor stage and it demonstrated that 


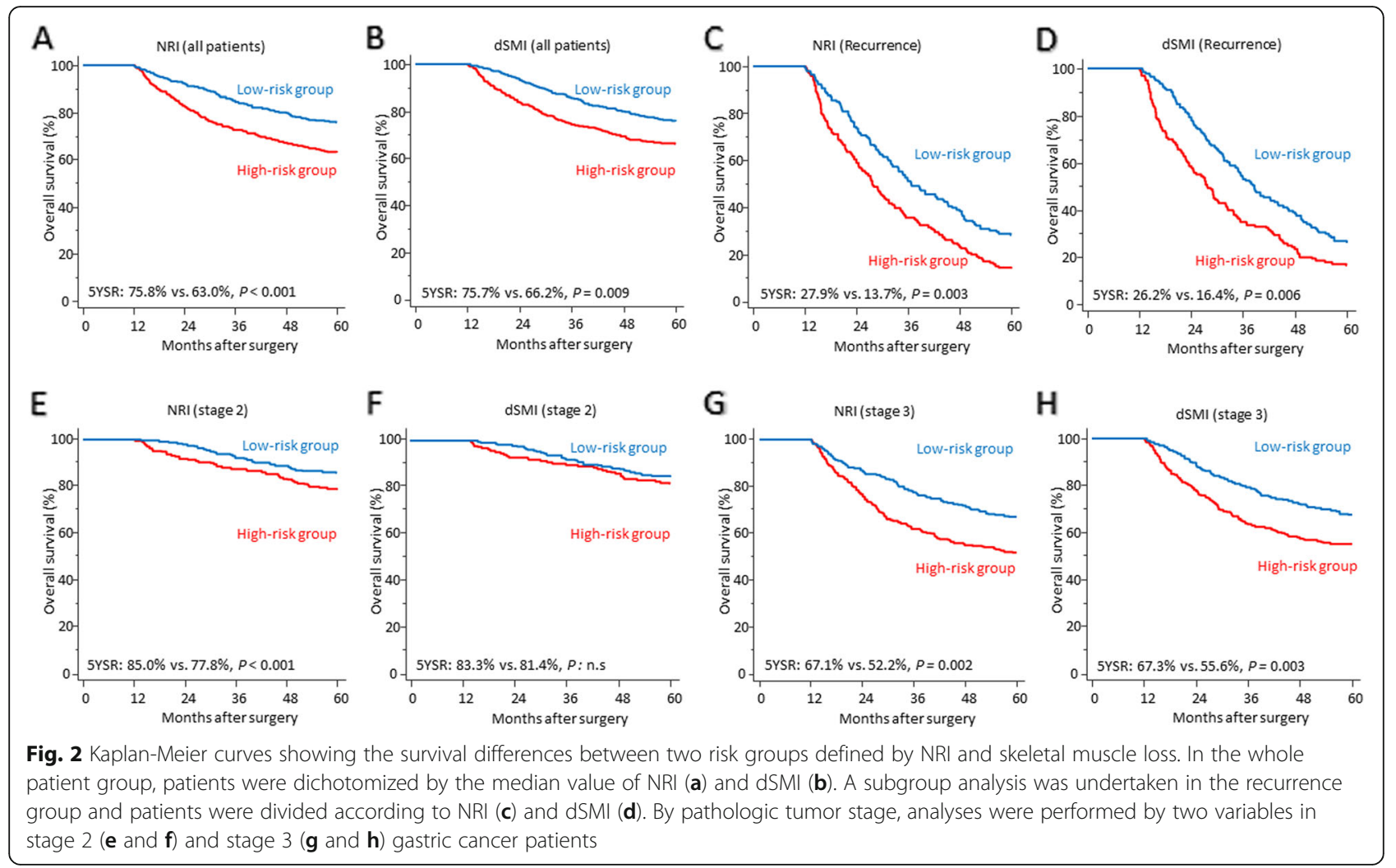

NRI stratified the prognosis in stage 2 group $(85.0 \%$ vs. $77.8 \%, P<0.001$ ) (Fig. 2e) but dSMI was not associated with the survival difference (Fig. 2f). However, we again ascertained that both factors significantly divided the survival curves of patients with stage 3 gastric cancer in line with Cox regression analysis (Fig. $2 \mathrm{~g}$ and $\mathrm{h}$ ).

Next, we evaluated two variables' performance in mortality prediction with a logistic regression model. For all patients with stage 2 and 3 gastric cancer, the accuracy to predict mortality resulted in the AUC of 0.61 (95\% CI: $0.58-0.64, P<0.001)$ and 0.54 (95\% CI: $0.51-0.57$,
$P=0.015)$ in NRI and dSMI, respectively. Then, we assessed a combined prediction model consisting of two factors and it exhibited the AUC of $0.63(P<0.001)$, which was higher than an individual variable. Subsequently, clinical factors including tumor recurrence, stage, and comorbidity index which were significant in multivariate analysis were combined to the prediction model, and the AUC was increased to 0.90 (95\% CI: $0.88-0.92$, sensitivity $79.6 \%$, specificity $91.9 \%, P<0.001$ ) (Fig. 3a). In the recurrence group, although the individual performance of NRI and dSMI was not significant, a
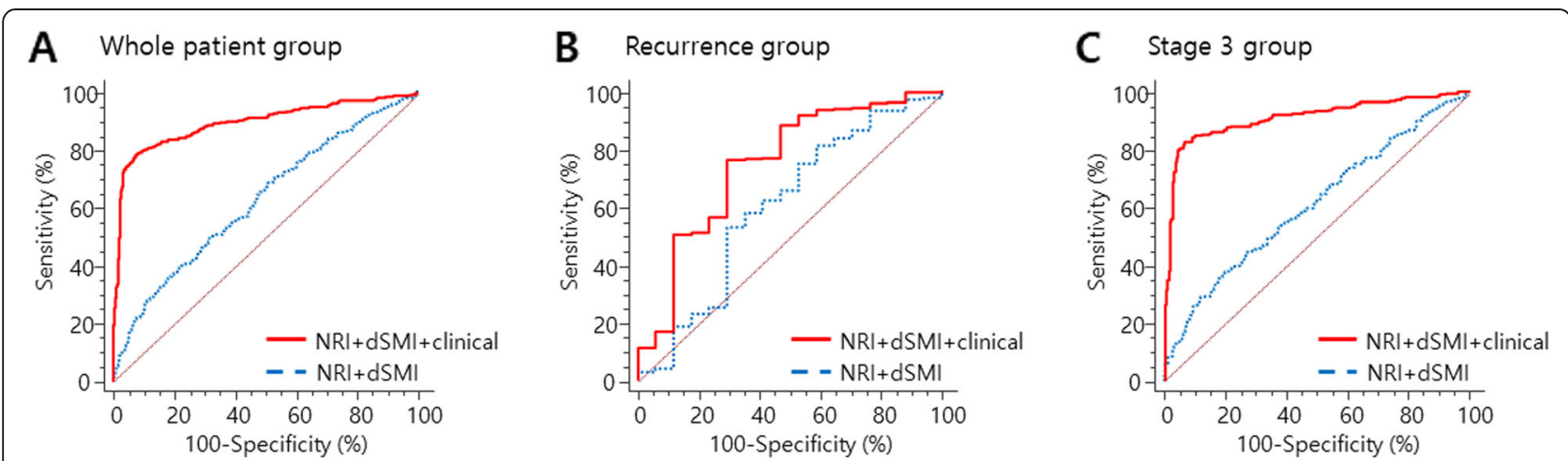

Fig. 3 Receiver operating characteristic (ROC) curves demonstrating the performance of preoperative NRI and dSMI in predicting mortality and the enhanced performance of prediction was seen when combined with clinical factors in the whole group (a), the recurrence group (b), and stage 3 gastric cancer patient group $(\mathbf{c})$ 
combined model with staging and Lauren's classification exhibited the AUC of 0.75 and it was statistically significant (95\% CI: $0.69-0.80$, sensitivity $76.3 \%$, specificity $70.6 \%, P=0.005$ ) (Fig. 3b). Finally, we evaluated the performance of two variables in stage 3 tumors and yielded the AUC of 0.61 but, when combined with recurrence and comorbidity index, the performance was improved to the AUC of 0.91 (95\% CI: $0.88-0.94$, sensitivity $82.8 \%$, specificity $93.5 \%, P<0.001$ ) (Fig. 3 c).

\section{Discussion}

In this study, the multivariate Cox-hazard regression results supported our hypothesis that the one-year loss of skeletal muscle after gastrectomy (dSMI) is a significant predictor of overall survival along with preoperative nutritional status (NRI) in gastric cancer patients who survived longer than 1 year. Although several powerful prognostic factors for overall survival including recurrence, TNM stage, and comorbidity (HRs, 16.838, 1.324, and 1.173 , respectively) were included in the multivariate analysis, the dSMI (HR 1.060) and preoperative NRI (HR 0.976) eventually remained as independent predictors. Kaplan-Meier curves also showed that less degree of postoperative muscle loss (small dSMI) and a better nutritional status before surgery (NRI) had a protective effect on the survival. These prognostic values of two variables were maintained even in patients with relapsed tumor and underscored in stage 3 gastric adenocarcinoma.

Nowadays, progressive loss of skeletal muscle mass has been highlighted as a prognostic factor in cancer patients, which is associated with cancer cachexia. Indeed, cachexia significantly contributes to mortality in patients with malignancy, accounting for more than $20 \%$ of cancer deaths [18]. Especially, in patients with gastric cancer, sarcopenia is known to be highly prevalent $[19,20]$, and a marked reduction in the initial body weight and muscle mass during the first postoperative year closely mimics the malnutrition and cancer cachexia cascade. Although several studies have reported preoperative sarcopenia as an indicator of poor prognosis, it could not reflect the prognostic uniqueness of the steep deterioration of muscle amount after gastrectomy [17, 21]. The increased mortality related to muscle loss in this study might be explained by that a higher degree of cachexia aggravates systemic inflammation and metabolic alterations leading to the poor prognosis in combination with a decrease in body protein stores. In addition, loss of muscle mass might also influence the tolerability of chemotherapy in those patients.

Our study, as a large-scale research, demonstrated that progressive muscle loss during the first year after gastrectomy is also a significant indicator of worse prognosis. Indeed, in a recent study based on a randomized multicenter trial, a marked loss in muscle or subcutaneous/visceral fat at 6 months after surgery could predict poor prognosis in patients with stage $2 / 3$ gastric cancer [22]. However, some limitations such as relatively small number of cases, inclusion of a significant number of patients without adjuvant treatment within the study cohort, and the absence of subgroup analyses alleviated the importance of the prognostic potential of muscle loss. To overcome these shortcomings, we confined our study group to patients undergoing adjuvant chemotherapy and performed several subgroup analyses by the presence of tumor recurrence and stage information. As a result, we were able to reveal the valuable prognostic relevance of NRI in stage 2 and 3 gastric cancers and muscle loss in stage 3 tumors.

As a nutritional factor, we adopted the NRI among various indices in this study because it consists of objective and easily measurable parameters used for follow-up of gastric cancer in outpatient settings and does not require additional measurement (e.g., triceps skin fold, detailed laboratory variable such as C-reactive protein, glycoprotein, prealbumin, and neutrophil/lymphocyte count). The preoperative NRI revealed its prognostic value in the recurrence group as well as whole patients. This result coincides with prior studies demonstrating that preoperative malnutrition could influence cancerrelated or -unrelated death in malignancies [23, 24]. However, the change in NRI between before and 1 year after surgery (dNRI) did not influence the overall survival in gastric cancer patients and the level of albumin was higher at postoperative 1 year compared to preoperative value. It might be attributed to an active educational program emphasizing high-protein diet to avoid dumping syndrome and to improve nutritional state after surgery, or enhanced adaptation of small intestine for protein absorption. However, our results should not hamper the importance of nutritional support for patients with gastric cancer.

Among several prognostic factors which were significant in this study, the dSMI and preoperative NRI could be valuable indicators because they may have potentials to improve prognosis through efforts to enhance the nutritional status before surgery or maintain the muscle mass with intensive exercise and nutritional support after gastrectomy. Recently, exercise and physical activities during cancer treatment has been greatly emphasized in various malignancies, and the therapeutic benefit of exercise interventions on cancer patients have been investigated [25-27]. In addition, in 2020, the American Cancer Society guidelines has been issued for diet and physical activity for cancer patients [28].

Only patients with stage 2 and 3 gastric cancer were included in the study because there is a discrepancy in treatment strategy and prognosis between stage 1 
tumors and more advanced cancers. The vast majority of patients with stage 1 gastric cancers are treated with surgery alone and have an excellent prognosis of 5-year overall survival rate reaching to $95 \%$. However, stage 2 and 3 cancers are indicated to surgery followed by chemotherapy, yielding the 3-year overall survival rate of $80.0 \%$, and a significant number of patients experience relapse despite of adjuvant treatment [29, 30]. In this perspective, the prognostic implication of body composition and nutrition becomes higher in stage 2 and 3 gastric cancer.

We acknowledge that this study has some limitations. First, although data were collected prospectively in the registry, this is a retrospective study based on data from a single institution. Second, as we excluded patients who died within 1 year postoperatively, the prognostic effect of skeletal muscle loss was applicable to patients who survived longer than 1 year after surgery. Third, we did not consider chemotherapy induced toxicity on the survival outcomes. Fourth, despite prognostic relevance, the therapeutic benefit of efforts to support nutrition and preserve muscle mass was not proven. Finally, as our study excluded patients treated with neoadjuvant strategy for advanced gastric cancer, clinical significance is limited in patients from Western countries. To overcome these limitations, a well-designed prospective multi-institutional study is required. Nevertheless, this study provides robust real-world evidence which is obtained from the large-scale study composed of 958 gastric cancer patients.

\section{Conclusions}

Preoperative NRI is a predictor of overall survival in stage 2 or 3 gastric cancer patients and skeletal muscle loss during the first postoperative year was significantly associated with the prognosis regardless of relapse in stage 3 tumors. These factors could be valuable adjuncts for accurate prediction of prognosis in gastric cancer patients.

\section{Supplementary Information}

The online version contains supplementary material available at https://doi. org/10.1186/s12885-021-07885-7.

Additional file 1 Supplementary Figure S1. Web-based toolkit for automatic segmentation of body composition. Supplementary Figure S2. Representative cases.

Additional file $\mathbf{2}$ Supplementary Table $\mathbf{S 1}$. Changes in body composition and nutritional parameters measured before and 1 year after surgery. Supplementary Table S2. Comparison of clinicopathologic characteristics of stage 2 and 3 gastric cancer patients based on NRI and dSMI.

\section{Abbreviations}

AUC: Area under curve; BMl: Body mass index; Cl: Confidence interval; CT: Computed tomography; HR: Hazard ratio; NRI: Nutritional risk index;
dNRI: difference in nutritional risk index; ROC: Receiver operating characteristic; SMA: Skeletal muscle area; SMI: Skeletal muscle index; dSMI: Difference in skeletal muscle index; SFA: Subcutaneous fat area; VFA: Visceral fat area

\section{Acknowledgements}

None.

\section{Authors' contributions}

KK: conception of the study, interpretation of data, creation of automated artificial intelligence software to assess muscle-fat measurement, drafting the work. KL: conception of the study, acquisition and interpretation of data, drafting the work. JL: conception of the study, analysis and interpretation of data. TP: acquisition of data imaging, analysis and interpretation of data. SK: acquisition of data imaging, analysis and interpretation of data. HJ: acquisition of data imaging, analysis and interpretation of data. CK: acquisition and analysis of data, drafting the work. JY: drafting the work, substantial revision. BK: drafting the work, substantial revision. IL: conception of the study, interpretation of data, drafting the work, substantial revision. All authors read and approved the final manuscript.

\section{Funding}

This study was supported by a grant from Asan Institute for Life Sciences and Corporate Relations of Asan Medical Center, Seoul, Korea (2017IF0761) and the Korea Health Industry Development Institute (No. HI18C1216). The funding bodies played no role in the design of the study and collection, analysis, and interpretation of data and in writing the manuscript.

\section{Availability of data and materials}

The datasets used and analysed during this study are available from the corresponding author on reasonable request.

\section{Ethics approval and consent to participate}

The protocol for this retrospective and registry-based cohort study followed the Helsinki declaration and was approved by the institutional review board of Asan Medical Center, Seoul, Korea (IRB No. 2017-0216). Informed consent was obtained in all participants in the study.

\section{Consent for publication}

Not applicable.

\section{Competing interests}

The authors declare that they have no competing interests.

\section{Author details}

${ }^{1}$ Department of Surgery, Gastric Cancer Center, Asan Medical Center, University of Ulsan College of Medicine, 88, Olympic-ro 43-gil, Songpa-gu, Seoul 05505, South Korea. ${ }^{2}$ Department of Radiology and Research Institute of Radiology, Asan Medical Center, University of Ulsan College of Medicine, Seoul, South Korea. ${ }^{3}$ Department of Nuclear Medicine, Asan Medical Center, University of Ulsan College of Medicine, Seoul, South Korea. ${ }^{4}$ Division of Biostatistics, Center for Medical Research and Information, Asan Medical Center, University of Ulsan College of Medicine, Seoul, South Korea. ${ }^{5}$ School of Computer Science and Engineering, Soongsil University, 369 Sangdo-Ro, Dongjak-Gu, Seoul, South Korea.

Received: 29 September 2020 Accepted: 8 February 2021

Published online: 12 February 2021

\section{References}

1. Bae JM, Park JW, Yang HK, Kim JP. Nutritional status of gastric cancer patients after total gastrectomy. World J Surg. 1998;22(3):254-60 discussion 60-1.

2. Braga M, Zuliani W, Foppa L, Di Carlo V, Cristallo M. Food intake and nutritional status after total gastrectomy: results of a nutritional follow-up. $\mathrm{Br}$ J Surg. 1988;75(5):477-80.

3. Lee $K$, Kim KW, Lee JB, Shin Y, Jang JK, Yook JH, et al. Impact of remnant stomach volume and anastomosis on nutrition and body composition in gastric cancer patients. Surg Oncol. 2019;31:75-82. 
4. Kim KH, Park DJ, Park YS, Ahn SH, Park DJ, Kim HH. Actual 5-year nutritional outcomes of patients with gastric Cancer. J Gastric Cancer. 2017;17(2):99_ 109.

5. Noh SH, Park SR, Yang HK, Chung HC, Chung IJ, Kim SW, et al. Adjuvant capecitabine plus oxaliplatin for gastric cancer after D2 gastrectomy (CLAS SIC): 5-year follow-up of an open-label, randomised phase 3 trial. Lancet Oncol. 2014;15(12):1389-96.

6. Migita K, Takayama T, Saeki K, Matsumoto S, Wakatsuki K, Enomoto K, et al. The prognostic nutritional index predicts long-term outcomes of gastric cancer patients independent of tumor stage. Ann Surg Oncol. 2013;20(8): 2647-54.

7. Lee S, Oh SY, Kim SH, Lee JH, Kim MC, Kim KH, et al. Prognostic significance of neutrophil lymphocyte ratio and platelet lymphocyte ratio in advanced gastric cancer patients treated with FOLFOX chemotherapy. BMC Cancer. 2013;13:350

8. Hacker UT, Hasenclever D, Linder N, Stocker G, Chung HC, Kang YK, et al. Prognostic role of body composition parameters in gastric/ gastroesophageal junction cancer patients from the EXPAND trial. J Cachexia Sarcopenia Muscle. 2020;11(1):135-44.

9. Z Zheng ZF, Lu J, Zheng CH, Li P, Xie JW, Wang JB, et al. A novel prognostic scoring system based on preoperative sarcopenia predicts the long-term outcome for patients after Ro resection for gastric Cancer: experiences of a high-volume center. Ann Surg Oncol. 2017;24(7):1795-803.

10. Moons KG, Altman DG, Reitsma JB, loannidis JP, Macaskill P, Steyerberg EW, et al. Transparent reporting of a multivariable prediction model for individual prognosis or diagnosis (TRIPOD): explanation and elaboration. Ann Intern Med. 2015;162(1):W1-73.

11. Edge SB, Compton CC. The American joint committee on Cancer: the 7th edition of the AJCC cancer staging manual and the future of TNM. Ann Surg Oncol. 2010;17(6):1471-4.

12. Charlson M, Szatrowski TP, Peterson J, Gold J. Validation of a combined comorbidity index. J Clin Epidemiol. 1994;47(11):1245-51.

13. Lauren $P$. The two histological Main types of gastric carcinoma: diffuse and so-called intestinal-type carcinoma. An attempt at a Histo-clinical classification. Acta Pathol Microbiol Scand. 1965;64:31-49.

14. Veterans Affairs Total Parenteral Nutrition Cooperative Study G. Perioperative total parenteral nutrition in surgical patients. N Engl J Med. 1991;325(8):525-32.

15. Japanese Gastric Cancer A. Japanese gastric cancer treatment guidelines 2014 (ver. 4). Gastric Cancer. 2017;20(1):1-19.

16. Park HJ, Shin Y, Park J, Kim H, Lee IS, Seo DW, et al. Development and validation of a deep learning system for segmentation of abdominal muscle and fat on computed tomography. Korean J Radiol. 2020;21(1):88-100.

17. Prado CM, Lieffers JR, McCargar LJ, Reiman T, Sawyer MB, Martin L, et al. Prevalence and clinical implications of sarcopenic obesity in patients with solid tumours of the respiratory and gastrointestinal tracts: a populationbased study. Lancet Oncol. 2008;9(7):629-35.

18. Fearon K, Strasser F, Anker SD, Bosaeus I, Bruera E, Fainsinger RL, et al. Definition and classification of cancer cachexia: an international consensus. Lancet Oncol. 2011;12(5):489-95.

19. Tan BH, Fearon KC. Cachexia: prevalence and impact in medicine. Curr Opin Clin Nutr Metab Care. 2008;11(4):400-7.

20. Tegels JJ, van Vugt JL, Reisinger KW, Hulsewe KW, Hoofwijk AG, Derikx JP, et al. Sarcopenia is highly prevalent in patients undergoing surgery for gastric cancer but not associated with worse outcomes. J Surg Oncol. 2015; 112(4):403-7.

21. Martin L, Birdsell L, Macdonald N, Reiman T, Clandinin MT, McCargar LJ et al. Cancer cachexia in the age of obesity: skeletal muscle depletion is a powerful prognostic factor, independent of body mass index. J Clin Oncol. 2013;31(12):1539-47

22. Park HS, Kim HS, Beom SH, Rha SY, Chung HC, Kim JH, et al. Marked loss of muscle, visceral fat, or subcutaneous fat after gastrectomy predicts poor survival in advanced gastric Cancer: single-center study from the CLASSIC trial. Ann Surg Oncol. 2018;25(11):3222-30.

23. Gallois C, Artru P, Lievre A, Auclin E, Lecomte T, Locher C, et al. Evaluation of two nutritional scores' association with systemic treatment toxicity and survival in metastatic colorectal cancer: an AGEO prospective multicentre study. Eur J Cancer. 2019;119:35-43.

24. Sakurai K, Tamura T, Toyokawa T, Amano R, Kubo N, Tanaka H, et al. Low preoperative prognostic nutritional index predicts poor survival post- gastrectomy in elderly patients with gastric Cancer. Ann Surg Oncol. 2016; 23(11):3669-76.

25. Yang J, Choi M, Choi J, Kang M, Jo A, Chung SH, et al. Supervised physical rehabilitation in the treatment of patients with advanced Cancer: a systematic review and meta-analysis. J Korean Med Sci. 2020;35(29):e242.

26. Tully R, Loughney L, Bolger J, Sorensen J, McAnena O, Collins CG, et al. The effect of a pre- and post-operative exercise programme versus standard care on physical fitness of patients with oesophageal and gastric cancer undergoing neoadjuvant treatment prior to surgery (the PERIOP-OG trial): study protocol for a randomised controlled trial. Trials. 2020;21(1):638.

27. Lee K, Zhou J, Norris MK, Chow C, Dieli-Conwright CM. Prehabilitative exercise for the enhancement of physical, psychosocial, and biological outcomes among patients diagnosed with Cancer. Curr Oncol Rep. 2020; 22(7):71.

28. Honda M, Hiki N, Kinoshita T, Yabusaki H, Abe T, Nunobe S, et al. Long-term outcomes of laparoscopic versus open surgery for clinical stage I gastric Cancer: the LOC-1 study. Ann Surg. 2016;264(2):214-22

29. Bang YJ, Kim YW, Yang HK, Chung HC, Park YK, Lee KH, et al. Adjuvant capecitabine and oxaliplatin for gastric cancer after D2 gastrectomy (CLAS SIC): a phase 3 open-label, randomised controlled trial. Lancet. 2012; 379(9813):315-21.

30. Sakuramoto S, Sasako M, Yamaguchi T, Kinoshita T, Fujii M, Nashimoto A, et al. Adjuvant chemotherapy for gastric cancer with S-1, an oral fluoropyrimidine. N Engl J Med. 2007;357(18):1810-20.

\section{Publisher's Note}

Springer Nature remains neutral with regard to jurisdictional claims in published maps and institutional affiliations.
Ready to submit your research? Choose BMC and benefit from:

- fast, convenient online submission

- thorough peer review by experienced researchers in your field

- rapid publication on acceptance

- support for research data, including large and complex data types

- gold Open Access which fosters wider collaboration and increased citations

- maximum visibility for your research: over $100 \mathrm{M}$ website views per year

At BMC, research is always in progress.

Learn more biomedcentral.com/submissions 